\title{
Quantity constrained equilibria
}

Citation for published version (APA):

Herings, P. J. J., van der Laan, G., \& Talman, A. J. J. (2001). Quantity constrained equilibria. METEOR, Maastricht University School of Business and Economics. METEOR Research Memorandum No. 023 https://doi.org/10.26481/umamet.2001023

Document status and date:

Published: 01/01/2001

DOI:

10.26481/umamet.2001023

Document Version:

Publisher's PDF, also known as Version of record

\section{Please check the document version of this publication:}

- A submitted manuscript is the version of the article upon submission and before peer-review. There can be important differences between the submitted version and the official published version of record.

People interested in the research are advised to contact the author for the final version of the publication, or visit the DOI to the publisher's website.

- The final author version and the galley proof are versions of the publication after peer review.

- The final published version features the final layout of the paper including the volume, issue and page numbers.

Link to publication

\footnotetext{
General rights rights.

- You may freely distribute the URL identifying the publication in the public portal. please follow below link for the End User Agreement:

www.umlib.nl/taverne-license

Take down policy

If you believe that this document breaches copyright please contact us at:

repository@maastrichtuniversity.nl

providing details and we will investigate your claim.
}

Copyright and moral rights for the publications made accessible in the public portal are retained by the authors and/or other copyright owners and it is a condition of accessing publications that users recognise and abide by the legal requirements associated with these

- Users may download and print one copy of any publication from the public portal for the purpose of private study or research.

- You may not further distribute the material or use it for any profit-making activity or commercial gain

If the publication is distributed under the terms of Article $25 \mathrm{fa}$ of the Dutch Copyright Act, indicated by the "Taverne" license above, 


\title{
Quantity Constrained Equilibria ${ }^{1}$
}

\author{
P. Jean-Jacques Herings ${ }^{2}$ Gerard van der $\operatorname{Laan}^{3} \quad$ Dolf Talman ${ }^{4}$
}

November 29, 2001

${ }^{1}$ This research is part of the research program "Competition and Cooperation".

${ }^{2}$ P.J.J. Herings, Department of Economics, Universiteit Maastricht, P.O. Box 616, 6200 MD Maastricht, The Netherlands, P.Herings@algec.unimaas.nl

${ }^{3}$ G. van der Laan, Department of Econometrics and Tinbergen Institute, Vrije Universiteit, De Boelelaan 1105, 1081 HV Amsterdam, The Netherlands, glaan@econ.vu.nl

${ }^{4}$ A.J.J. Talman, Department of Econometrics \& Operations Research and CentER, Tilburg University, P.O. Box 90153, 5000 LE Tilburg, The Netherlands, talman@kub.nl 


\begin{abstract}
In a standard general equilibrium model it is assumed that there are no price restictions and that prices adjust infinitely fast to their equilibrium values. Price rigidities may cause that a competitive equilibrium cannot be attained, and rationing on net demands or supplies is needed to clear the markets. Bénassy, Drèze and Younès proved in the mid 1970s that there exist equilibria with rationing. Moreover, rationing is such that not both demand and supply of a good are rationed simultaneously, at least one commodity is not rationed at all, and there is rationing on the net supply or net demand of a good only if the price of that good is on its lower or upper bound, respectively. In the 1980s disequilibrium models with only rationing on net supplies were introduced. In all these models prices are restricted by positive lower and upper bounds.

In this paper the set of admissible prices is allowed to be an arbitrary convex set. For such an arbitrary set it cannot be guaranteed that there exists a constrained equilibrium satisfying that a price will be on its upper or lower bound in case of rationing. We introduce a more general equilibrium concept, called Quantity Constrained Equilibrium (QCE). At such an equilibrium the levels of supply and demand rationing are completely determined by the components of a direction in which the price system cannot be moved further without leaving the set of admissible prices. When the set is compact, we show the existence of a connected set of QCEs, containing two trivial no-trade equilibria. Moreover, the set contains for every commodity a generalized Drèze equilibrium, being a $\mathrm{QCE}$ at which this commodity is not being rationed, and also a generalized supply-constrained equilibrium without demand rationing. We apply this main result to several special cases, including the case of an unbounded set of admissible prices.
\end{abstract}

Key words: exchange economy, price rigidities, equilibrium, rationing.

JEL-code: C62, C63, C68, D51. 


\section{Introduction}

Perfect competition is a basic assumption in economic theory. All agents are assumed to be price takers and therefore express their demand and supply at the prevailing prices on the market. Trade takes place only at a price system for which for every commodity total demand equals total supply. It is assumed that there is no restriction on the prices and that prices adjust infinitely fast. Walras (1874) considered the problem of the existence of a general competitive equilibrium, corresponding to a price system at which all markets clear simultaneously. Under very general conditions the existence of such a Walrasian equilibrium has been shown by Arrow and Debreu (1954).

Unemployment and excess supply on commodity and factor markets are apparently serious problems in many countries. The literature has identified a variety of reasons why prices might be prevented from adjusting to Walrasian values. Politically desired price restrictions and regulations, such as price controls to reduce inflation, minimum wages, or price indexation are mentioned by Cox (1980), Nguyen and Whalley (1986, 1990), Ginsburgh and Van der Heyden (1988), Herings (1997) and Tuinstra (2000). Bénassy (1993) considers non-competitive price systems resulting from models with imperfect competition. When markets are incomplete, price regulations may be used to generate Pareto improvements as stressed by Drèze and Gollier (1993), Herings and Polemarchakis (2000), and Drèze (2001).

When trade occurs against disequilibrium prices, markets clear through quantity adjustments, e.g. by imposing quantity rationing, queuing systems or production quota. In the mid 1970s Bénassy (1975), Drèze (1975) and Younès (1975) independently developed market clearing mechanisms for economies with price restrictions by using quantity rationing. In that approach, an agent chooses a most preferred consumption bundle, subject to both a budget constraint and quantity constraints on net demands and supplies. To express the transparency of markets, quantity rationing is not allowed to affect excess supply and excess demand simultaneously. When price rigidities are formulated by lower and upper bounds on the prices of the commodities, Drèze (1975) proved the existence of an equilibrium at which at least one a priori chosen commodity is not being rationed at all. Further supply (demand) rationing on a commodity can only be binding when its price is on its lower (upper) bound. Such an equilibrium is called a Drèze equilibrium.

In the 1980s both van der Laan and Kurz argued that in practice rationing on net demand is rarely observed and difficult to implement. This motivated these authors to consider equilibria with only rationing on the net supplies. In van der Laan (1980, 1982) and Kurz (1982) the existence of an equilibrium with only rationing on the net supplies and at least one commodity not rationed at all has been proven. Such an equilibrium is called a supply-constrained or unemployment equilibrium. 
In Herings (1998) the entire set of equilibria, called constrained equilibria, has been characterized. There exists a connected set of constrained equilibria, containing two trivial equilibria. In one trivial equilibrium, the trivial supply-constrained equilibrium, all commodities are fully rationed in their supply and all prices are on their lower bound, whereas at the other trivial equilibrium, the trivial demand-constrained equilibrium, all commodities are fully rationed in their demand and all prices are on their upper bound. The connected set also contains for every commodity a constrained equilibrium at which that commodity is not being rationed at all. In particular, this implies the existence of a Drèze equilibrium. Moreover, one of these equilibria is a supply-constrained equilibrium and another one a demand-constrained equilibrium. It is possible to compute this connected set of equilibria by using the algorithm presented in Herings, Talman and Yang (1996). For an interpretation of these results in terms of self-fulfilling coordination failures, as well as for an extension to economies with production, see Drèze (1997) and Citanna, Crès, Drèze, Herings and Villanacci (2001).

In Dehez and Drèze (1984) and van der Laan (1984), economies with price indexations are considered. It has been shown that there exists a supply-constrained equilibrium without rationing on the numeraire commodity, when the set of feasible prices allows for enough flexibility of the price level of the non-numeraire commodities. Weddepohl (1987) discusses extensively linkages between prices through index functions and shows that a supply constrained equilibrium in which at least one commodity is not rationed exists if the system of indexes is non-circular, i.e. no commodity is directly or indirectly indexed by itself. In these papers it is also argued that the equilibrium condition that rationing of a commodity is not allowed unless its price restriction is binding, cannot be maintained when prices are tied to each other through index functions.

In this paper we consider the general case where price restrictions lead to an arbitrary convex set of nonnegative prices. This set could be a point as in the fix-price literature, a cube with lower and upper bounds for the prices as in the model of Drèze (1975), a slice of a convex cone in case of price indexation as in the models of Dehez and Drèze (1984) and Weddepohl (1987). For the general case it cannot be assured that there is only rationing for a commodity if its price is equal to its minimum or maximum value. Therefore we generalize the equilibrium concept to the one of Quantity Constrained Equilibrium. At such an equilibrium, commodities may be rationed only in case the price system lies on the boundary of the set of admissible prices. Rationing is determined by a direction in which the prices cannot adjust, i.e. by a vector in the normal cone of the set of admissible prices. A negative component of this vector induces supply rationing on the related commodity, and, likewise, a positive component induces demand rationing.

We will show that in case the set of prices is compact and only contains positive price 
vectors, there exists a connected set of Quantity Constrained Equilibria (QCE) containing two trivial equilibria, a trivial supply-constrained equilibrium with full rationing on the supply of all commodities and prices such that the value of the total initial endowments is minimized, and a trivial demand-constrained equilibrium where all commodities are fully rationed in their demand and the value of the total initial endowments is maximized. The connected set also contains for every commodity a generalized Drèze equilibrium, being a QCE at which that commodity is not being rationed at all, a generalized supplyconstrained equilibrium, at which there is no demand rationing and at least one commodity is not rationed at all, and a generalized demand-constrained equilibrium, at which there is no supply rationing and at least one commodity is not rationed at all. This existence result is proved by using the fixed point theorem of Browder (1960). The main result is applied to several special cases.

We also consider cases in which the set of admissible prices is not bounded or contains zero prices, allowing for models in which some prices can vary freely and others are tied by index systems. In case the set of prices is unbounded, the connected set of QCEs is also unbounded with some of the prices going to infinity, while simultaneously for any commodity with price bounded from above, its relative price tends to zero and eventually the commodity becomes fully constrained in its demand, implying no trade for this commodity. In the limit the economy reduces to an economy with trade only in the commodities with prices unbounded from above. When the prices of these commodities are not tied to prices of other commodities, these prices tend to Walrasian equilibrium values for this reduced economy. In case this holds for all commodities, there is a connected set of QCEs leading from the trivial full supply constrained equilibrium to a Walrasian equilibrium without rationing.

The paper has been organized as follows. Section 2 describes the model and introduces the general concept of Quantity Constrained Equilibrium. For the compact, convex case with positive prices the existence results are given in Section 3. Specific cases are discussed in Section 4, as well as extensions, including the unbounded case.

\section{The model}

We consider an exchange economy $\mathcal{E}=\left(\left\{\mathcal{X}^{i}, \succeq^{i}, w^{i}\right\}_{i=1}^{m}, P\right)$. In this economy there are $m$ consumers, indexed $i=1, \ldots, m$, and $n$ commodities, indexed $j=1, \ldots, n$. For $k$ a positive integer, we denote $I_{k}=\{1, \ldots, k\}$. Each consumer $i \in I_{m}$ is characterized by a consumption set $\mathcal{X}^{i}$, a preference preordering $\succeq^{i}$ on $\mathcal{X}^{i}$, and a vector of initial endowments $w^{i}$. The total endowment $w$ is defined by $w=\sum_{i \in I_{m}} w^{i}$. We assume that the admissible price systems in the economy $\mathcal{E}$ are described by the set $P \subset \mathbb{R}_{+}^{n}$. The following standard 
assumptions $\mathrm{X}, \mathrm{U}$ and $\mathrm{W}$ with respect to the economy $\mathcal{E}$ are made.

\section{Assumption X}

For every consumer $i \in I_{m}$, the consumption set $\mathcal{X}^{i}$ is a closed and convex subset of $\mathbb{R}_{+}^{n}$ and $\mathcal{X}^{i}+\mathbb{R}_{+}^{n} \subset \mathcal{X}^{i}$.

\section{Assumption U}

For every consumer $i \in I_{m}$, the preference preordering $\succeq^{i}$ on $\mathcal{X}^{i}$ is complete, continuous, strongly monotonic, and strictly convex. ${ }^{1}$

\section{Assumption W}

For every consumer $i \in I_{m}$, the vector of initial endowments $w^{i}$ belongs to the interior of $\mathcal{X}^{i}$.

The assumption of strict convexity allows us to work with demand functions instead of demand correspondences and thereby simplifies our notation. All our results carry over to the case of convex preferences. Also the assumption of strongly monotonic preferences is made for the sake of simplicity and can be relaxed considerably.

The set $P$ of admissible prices may or may not contain a Walrasian price system for the economy $\mathcal{E}$. In general this is not the case, and one needs an equilibrium concept involving vectors of quantity constraints on the net demands and the net supplies of the commodities. In this paper, we analyze the case of uniform rationing systems, meaning that rationing constraints are the same for all households. Our results carry over to a variety of other rationing systems, see Herings (1996a) for a general treatment of rationing systems.

Given a price system $p \in P$, a rationing scheme on supply $\ell \in-\mathbb{R}_{+}^{n}$, and a rationing scheme on demand $u \in \mathbb{R}_{+}^{n}$, the constrained budget set of consumer $i \in I_{m}$ is given by

$$
B^{i}(p, \ell, u)=\left\{x^{i} \in \mathcal{X}^{i} \mid p \cdot x^{i} \leq p \cdot w^{i} \text { and } \ell_{k} \leq x_{k}^{i}-w_{k}^{i} \leq u_{k}, \forall k \in I_{n}\right\} .
$$

The number $\ell_{k}\left(u_{k}\right)$ is the net amount of commodity $k$ that a consumer can sell (buy) at most. These constraints are determined endogenously and serve to equilibrate markets when, because of price restrictions, the price mechanism is not capable of doing so.

The corresponding constrained demand $d^{i}(p, \ell, u)$ of consumer $i$ is defined as the best element for $\succeq^{i}$ in $B^{i}(p, \ell, u)$. Because of the strict convexity and strong monotonicity assumptions, this element is unique and lies on the budget hyperplane, i.e. $p \cdot d^{i}(p, \ell, u)=$ $p \cdot w^{i}$

\footnotetext{
${ }^{1}$ A preference preordering $\succeq^{i}$ is said to be strongly monotonic if $\bar{x}^{i}, \widehat{x}^{i} \in \mathcal{X}^{i}, \bar{x}^{i} \leq \widehat{x}^{i}$, and $\bar{x}^{i} \neq \widehat{x}^{i}$ implies $\widehat{x}^{i} \succ^{i} \bar{x}^{i}$. A preference preordering $\succeq^{i}$ is said to be strictly convex when for any pair $\bar{x}^{i}, \widehat{x}^{i} \in \mathcal{X}^{i}$, such that $\bar{x}^{i} \neq \widehat{x}^{i}, \bar{x}^{i} \sim^{i} \widehat{x}^{i}$, it holds that $\lambda \bar{x}^{i}+(1-\lambda) \widehat{x}^{i} \succ^{i} \bar{x}^{i}$ for $\lambda \in(0,1)$.
} 
A well-known case discussed in the literature is when the set $P$ is given by

$$
C^{n}=\left\{p \in \mathbb{R}_{+}^{n} \underline{p}_{k} \leq p_{k} \leq \bar{p}_{k}, k \in I_{n}\right\}
$$

where $\underline{p}_{k}$ and $\bar{p}_{k}$ are a priori given lower and upper bounds for the price $p_{k}$ of good $k \in I_{n}$, satisfying $0<\underline{p}_{k} \leq \bar{p}_{k}$. In Drèze [10], a rationed equilibrium with respect to such a cubic set of admissible price systems and a uniform rationing system is defined as follows.

\section{Definition 2.1 (Constrained Equilibrium)}

$A$ Constrained Equilibrium for the economy $\mathcal{E}=\left(\left\{\mathcal{X}^{i}, \succeq^{i}, w^{i}\right\}_{i=1}^{m}, C^{n}\right)$ is a price system $p^{*} \in C^{n}$, a rationing scheme $\left(\ell^{*}, u^{*}\right) \in-\mathbb{R}_{+}^{n} \times \mathbb{R}_{+}^{n}$, and, for every consumer $i \in I_{m}$, a consumption bundle $x^{* i} \in \mathcal{X}^{i}$ such that

(i) for all $i \in I_{m}, x^{* i}=d^{i}\left(p^{*}, \ell^{*}, u^{*}\right)$;

(ii) $\quad \sum_{i=1}^{m} x^{* i}=w$;

(iii) for all $k \in I_{n}: x_{k}^{* h}-w_{k}^{h}=\ell_{k}^{*}$ for some $h \in I_{m}$ implies $x_{k}^{* i}-w_{k}^{i}<u_{k}^{*}, \forall i \in I_{m}$, and, analogously, $x_{k}^{* h}-w_{k}^{h}=u_{k}^{*}$ for some $h \in I_{m}$ implies $x_{k}^{* i}-w_{k}^{i}>\ell_{k}^{*}, \forall i \in I_{m}$;

(iv) for all $k \in I_{n}$, if there is $i \in I_{m}$ such that $\ell_{k}^{*}=x_{k}^{* i}-w_{k}^{i}$, then $p_{k}^{*}=\underline{p}_{k}$ and if there is $i \in I_{m}$ such that $u_{k}^{*}=x_{k}^{* i}-w_{k}^{i}$, then $p_{k}^{*}=\bar{p}_{k}$.

Condition (i) requires that the consumption of each consumer equals his constrained demand, while Condition (ii) is the market clearing condition. Condition (iii) implies that there is not simultaneously rationing on both sides of a market. Condition (iv) precludes supply rationing in the market of some commodity as long as its price is not on its lower bound, whereas demand rationing in the market of a commodity does not take place if its price is not on its upper bound. Observe that there exist two trivial equilibria. One is the trivial supply-constrained equilibrium, with full rationing on the supply of all commodities, so $\ell^{*}=0^{n}$, price system $p^{*}=\underline{p}$ and allocation $x^{* i}=w^{i}, i \in I_{m}$. The other is the trivial demand-constrained equilibrium, with full rationing on the demand of all commodities, so $u^{*}=0^{n}$, price system $p^{*}=\bar{p}$ and allocation $x^{* i}=w^{i}, i \in I_{m}$.

It has been shown in Herings (1998) that there exists a connected set of constrained equilibria that contains both trivial equilibria. This result admits very simple proofs of a number of special cases that were treated in the literature before, like the existence of a Drèze equilibrium with respect to commodity $k$ (Drèze (1975)) and the existence of a supply-constrained equilibrium (van der Laan (1980, 1982)). A Drèze equilibrium with respect to commodity $k$ is a constrained equilibrium without rationing in the market for commodity $k$. A supply-constrained equilibrium is a constrained equilibrium without rationing on the demand, and without rationing on the market of at least one commodity. 
An algorithm to compute the connected set of constrained equilibria has been proposed in Herings, Talman and Yang (1996).

Constrained equilibria with different rationing schemes may well yield the same constrained equilibrium allocation. When there is no rationing on the supply side of a market, the precise specification of $\ell_{k}^{*}$ is immaterial, as long as $\ell_{k}^{*}<x_{k}^{* i}-w_{k}^{i}$ for all $i \in I_{m}$. Analogously, when there is no rationing on the demand side of a market, any rationing scheme $u_{k}^{*}$ satisfying $u_{k}^{*}>x_{k}^{* i}-w_{k}^{i}$ for all $i \in I_{m}$ is compatible with a constrained equilibrium. The freedom in the specification of a non-binding rationing scheme can be used to simplify notation and proofs by making a particular choice for a non-binding rationing scheme. We focus on rationing schemes $(l, u)$ that are represented by a single vector $r \in[-w, w]$, called the rationing vector, where $[-w, w]=\left\{x \in \mathbb{R}^{n} \mid-w \leq x \leq w\right\}$. A rationing vector $r \in[-w, w]$ induces a rationing scheme $(l, u)$ given by

$$
l=-w-r \leq 0^{n} \text { and } u=w-r \geq 0^{n} .
$$

We modify the definition of a constrained equilibrium as follows.

\section{Definition 2.2 (Constrained Equilibrium)}

$A$ Constrained Equilibrium for the economy $\mathcal{E}=\left(\left\{\mathcal{X}^{i}, \succeq^{i}, w^{i}\right\}_{i=1}^{m}, C^{n}\right)$ is a price system $p^{*} \in C^{n}$, a rationing vector $r^{*} \in[-w, w]$, and, for every consumer $i \in I_{m}$, a consumption bundle $x^{* i} \in \mathcal{X}^{i}$ such that

(i) for all $i \in I_{m}, x^{* i}=d^{i}\left(p^{*},-w-r^{*}, w-r^{*}\right)$;

(ii) $\quad \sum_{i=1}^{m} x^{* i}=w$;

(iii) for all $k \in I_{n}$, if there is $i \in I_{m}$ such that $-w_{k}-r_{k}^{*}=x_{k}^{* i}-w_{k}^{i}$, then $p_{k}^{*}=\underline{p}_{k}$ and if there is $i \in I_{m}$ such that $w_{k}-r_{k}^{*}=x_{k}^{* i}-w_{k}^{i}$, then $p_{k}^{*}=\bar{p}_{k}$.

Since $0^{n} \leq x^{* i} \leq w$ and $0^{n}<w^{i}<w$ and therefore $-w<x^{* i}<w$ for any $i$, a negative value of $r_{k}^{*}$ implies that there can be only rationing on the supply of commodity $k$, while the opposite holds in case of a positive value of $r_{k}^{*}$. When $r_{k}^{*}=0$, there can be neither rationing on the demand side nor on the supply side of the market for commodity $k$. Therefore Condition (iii) replaces both Condition (iii) and Condition (iv) of Definition 2.1.

In this paper we allow for a general set $P$ of admissible prices, for instance when some of the prices are tied to prices of other commodities through indexation. Because in such cases $P$ may not be a cube, and thus there may be no independently given lower and upper bounds for the prices, we have to modify the complementarity Condition (iii) of Definition 2.2. For some special cases this issue was raised already by Chetty and Nayak 
(1978), Kurz (1982), Dehez and Drèze (1984), van der Laan (1984) and Weddepohl (1987). As a simple example, consider the set $P$ given by

$$
P=\left\{p \in \mathbb{R}_{+}^{n} \mid p_{1} \geq 0, \sum_{j \in I} p_{j}=1, \underline{p}_{j} \leq p_{j} \leq \bar{p}_{j}, j \in J\right\},
$$

where $I \subset I_{n} \backslash\{1\}$ is a set of index commodities and $J=I_{n} \backslash(I \cup\{1\})$ is the set of indexed commodities. Commodity 1 is the numeraire commodity. In van der Laan (1984) and Weddepohl (1987), it has been shown that there exists a supply-constrained equilibrium without rationing on the numeraire commodity, while simultaneously all commodities in $I$ are unrationed or at least one of the commodities in $J$ is unrationed. In the latter case, Condition (iii) of Definition 2.2 is not satisfied. In general there is rationing on all commodities in $I$, although because of the fact that $\sum_{j \in I} p_{j}=1$, at least one of the prices $p_{j}, j \in I$, is positive and is not downwards rigid. It has been proved that in this case there exists a constrained equilibrium such that the level of rationing on each of these commodities is given by the same fraction of the initial endowment.

An equilibrium concept for general price restrictions should contain the concept of Definition 2.2 as a special case and should also deal with a set of prices as in (1) in a satisfactory way. In our equilibrium concept we require that rationing is not allowed when the equilibrium price vector $p^{*}$ lies in the interior of the set $P$, because then the price system can move freely into any direction and $p^{*}$ is a Walrasian equilibrium price vector. In case in equilibrium the price vector $p^{*}$ does not lie in the interior of the set $P$, which is always the case when $P$ is not full-dimensional, there are vectors that point outwards to $P$ at $p^{*}$ and which are directions in which the price system cannot move without leaving the set of admissible prices $P$. The collection of all these directions forms the normal cone at $p^{*}$ to $P$. For any $p^{*} \in P$, the normal cone $G\left(p^{*}\right)$ to $P$ at $p^{*}$ is given by

$$
G\left(p^{*}\right)=\left\{r \in \mathbb{R}^{n} \mid p \cdot r \leq p^{*} \cdot r \text { for any } p \in P\right\} .
$$

The normal cone gives information on the directions in which the price system is restricted to move, and therefore on the kind of rationing that occurs in equilibrium. In our equilibrium concept we require that in equilibrium the normal cone $G\left(p^{*}\right)$ contains an element $r^{*} \in[-w, w]$ which completely determines the levels of supply and demand rationing. When $r_{j}^{*}$ is positive (negative), in which case the price of commodity $j$ cannot be increased (decreased) without leaving $P$ when moving in the direction $r^{*}$, we allow only for demand (supply) rationing on commodity $j$. The level of rationing is determined by the value of $r_{j}^{*}$. The higher the absolute value of $r_{j}^{*}$, the tighter the rationing will be. When $r_{j}^{*}=0$, in which case the price of commodity $j$ is not changing when moving in the direction of $r^{*}$, no rationing is allowed on commodity $j$. This gives us the following definition of the general concept of a Quantity Constrained Equilibrium. 


\section{Definition 2.3 (Quantity Constrained Equilibrium)}

$A$ Quantity Constrained Equilibrium (QCE) for the economy $\mathcal{E}=\left(\left\{\mathcal{X}^{i}, \succeq^{i}, w^{i}\right\}_{i=1}^{m}, P\right)$ is a price system $p^{*} \in P$, a rationing vector $r^{*} \in[-w, w]$, and, for every consumer $i \in I_{m}$, a consumption bundle $x^{* i} \in \mathcal{X}^{i}$ satisfying

(i) for all $i \in I_{m}, x^{* i}=d^{i}\left(p^{*},-w-r^{*}, w-r^{*}\right)$;

(ii) $\quad \sum_{i=1}^{m} x^{* i}=w$;

(iii) $r^{*} \in G\left(p^{*}\right)$.

As motivated above, Condition (iii) links the rationing vector to the price restrictions. The rationing scheme is completely determined by a vector $r^{*}$ in the normal cone $G\left(p^{*}\right)$ at the equilibrium price $p^{*}$.

Since $0 \leq x^{* i} \leq w$ and $0<w^{i}<w$, it holds that in a QCE $-w_{k}<x_{k}^{* i}-w_{k}^{i}<w_{k}$ for all $k \in I_{n}$ and $i \in I_{m}$. Hence, in a QCE, a consumer $i \in I_{m}$ can only be rationed in his demand for good $k$ if $r_{k}^{*}=w_{k}-\left(x_{k}^{* i}-w_{k}^{i}\right)>0$. Analogously, some consumer $i \in I_{m}$ can only be rationed in his supply of good $k$ if $r_{k}^{*}=-w_{k}-\left(x_{k}^{* i}-w_{k}^{i}\right)<0$. Hence there cannot be rationing simultaneosly on both sides of any market. Notice that the rationing on the supply (demand) of commodity $k$ is tighter, the closer $r_{k}^{*}$ is to $-w_{k}$ ( $w_{k}$, respectively). In particular, there is full supply rationing if $r_{k}^{*}=-w_{k}$ and there is full demand rationing if $r_{k}^{*}=w_{k}$. When $p^{*}$ is in the interior of $P$, then $r^{*}=0^{n}$, in which case the rationing scheme on demand equals $w$ and the rationing scheme on supply $-w$, implying that none of the rationing constraints can be binding.

Summarizing, in a QCE consumers maximize their utility in their constrained budget sets, total demand equals total supply, there is no simultaneous rationing on both supply and demand, and a vector in the normal cone to $P$ determines the tightness of rationing. In case $P=C^{n}, r_{k}^{*}<0$ implies $p_{k}^{*}=\underline{p}_{k}$ and $r_{k}^{*}>0$ implies $p_{k}^{*}=\bar{p}_{k}$, yielding precisely Condition (iii) of Definition 2.2.

For the set $P$ given in (1), it holds for any positive price vector $p$ and any $r \in G(p)$ that $r_{k}=r_{h}$ for every two indices $k$ and $h$ in the index set $I$. Hence, if there is rationing on these commodities, then according to Condition (iii) of Definition 2.3, the amount of rationing is the same for all commodities in $I$.

From an economic point of view, it is of crucial importance that our equilibrium concept is independent of the units of measurement that are used in the definition of a commodity. Suppose that the unit of measurement used in the definition of commodity $k$ is multiplied by a positive constant $\alpha_{k}, k=1, \ldots, n$. An economy $\mathcal{E}(\alpha)$ with initial endowments $w^{i}(\alpha)$ given by $w_{k}^{i}(\alpha)=w_{k} / \alpha_{k}, k=1, \ldots, n$, set of admissible prices

$$
P(\alpha)=\left\{\widehat{p} \in \mathbb{R}^{n} \mid \widehat{p}_{k}=\alpha_{k} p_{k}, k=1, \ldots, n, \text { for some } p \in P\right\}
$$


and appropriately redefined consumption sets $\mathcal{X}^{i}(\alpha)$ and preference relations $\succeq^{i}(\alpha)$ should have an equivalent set of QCEs as the economy $\mathcal{E}$. More precisely, for each QCE $\left(p^{*}, r^{*}, x^{*}\right)$ of $\mathcal{E}$ there should be a $\operatorname{QCE}\left(p^{*}(\alpha), r^{*}(\alpha), x^{*}(\alpha)\right)$ of $\mathcal{E}(\alpha)$ and vice versa, where $p^{*}(\alpha)$ is obtained from $p^{*}$ by componentwise multiplication by $\alpha$, and $r^{*}(\alpha)$ and $x^{*}(\alpha)$ are obtained from $r^{*}$ and $x^{*}$ by componentwise division by $\alpha$. The following result claims that equivalence holds.

\section{Theorem 2.4}

The set of equilibria of $\mathcal{E}=\left(\left\{\mathcal{X}^{i}, \succeq^{i}, w^{i}\right\}_{i=1}^{m}, P\right)$ is equivalent to the set of equilibria of $\mathcal{E}(\alpha)=\left(\left\{\mathcal{X}^{i}(\alpha), \succeq^{i}(\alpha), w^{i}(\alpha)\right\}_{i=1}^{m}, P(\alpha)\right)$ for any choice of $\alpha \gg 0$.

\section{Proof.}

It is obvious that $\left(p^{*}, r^{*}, x^{*}\right)$ satisfies Conditions (i) and (ii) of Definition 2.3 of a QCE of $\mathcal{E}$ if and only if $\left(p^{*}(\alpha), r^{*}(\alpha), x^{*}(\alpha)\right)$ satisfies Conditions (i) and (ii) of Definition 2.3 of a $\mathrm{QCE}$ of $\mathcal{E}(\alpha)$. It is easily verified that $r^{*} \in G\left(p^{*}\right)$ if and only if $r^{*}(\alpha) \in G^{\alpha}\left(p^{*}(\alpha)\right)$, where $G^{\alpha}$ denotes the normal cone at $P(\alpha)$. This shows that $\left(p^{*}, r^{*}, x^{*}\right)$ satisfies Condition (iii) of the definition of a QCE of $\mathcal{E}$ if and only if $\left(p^{*}(\alpha), r^{*}(\alpha), x^{*}(\alpha)\right)$ satisfies Condition (iii) of the definition of a $\mathrm{QCE}$ of $\mathcal{E}(\alpha)$.

Q.E.D.

\section{Existence results}

To consider the existence of QCEs, in this section we restrict ourselves to the case that $P$ satisfies the following Assumption P. In the next section we will also allow for zero prices and an unbounded set of admissible prices.

\section{Assumption P}

The set $P$ of admissible prices is a non-empty, convex and compact subset in the interior of $\mathbb{R}_{+}^{n}$.

We first show that there are two trivial no-trade QCEs with full rationing on the supply, respectively the demand. Let $P^{0}$ and $P^{1}$ be given by

$$
P^{0}=\{p \in P \mid w \cdot p \leq w \cdot \widetilde{p} \text { for all } \widetilde{p} \in P\}
$$

and

$$
P^{1}=\{p \in P \mid w \cdot p \geq w \cdot \widetilde{p} \text { for all } \widetilde{p} \in P\} .
$$

Notice that the intersection of $P^{0}$ and $P^{1}$ is either empty or equal to $P$. Prices in $P^{0}$ are such that the value of total income $p \cdot w$ is minimized, and prices in $P^{1}$ such that this 
value is maximized. The next result claims that there is a trivial no-trade QCE with full rationing on the supply at any price in $P^{0}$ and a trivial no-trade QCE with full rationing on the demand at any price in $P^{1}$.

\section{Theorem 3.1}

For any $p \in P^{0}$ there is a trivial supply-constrained equilibrium with rationing vector $r^{*}=-w$ and allocation $x^{* i}=w^{i}, i \in I_{m}$. For any $p \in P^{1}$ there is a trivial demandconstrained equilibrium with rationing vector $r^{*}=w$ and allocation $x^{* i}=w^{i}, i \in I_{m}$.

\section{Proof.}

Take any $p \in P^{0}$. Clearly, $p \in \operatorname{bnd}(P)$. By definition of $P^{0}$ we have that $-w \in G(p)$. Taking $r=-w$ gives for any $i \in I_{m}$ that $B^{i}(p, 0,2 w)=\left\{w^{i}\right\}$ and hence $d^{i}(p, 0,2 w)=w^{i}$, implying that markets clear and thus a no-trade equilibrium with full rationing on supply is obtained.

Analogously, take any $p \in P^{1}$. It holds that $w \in G(p)$. We have for any $i \in I_{m}$ that $B^{i}(p,-2 w, 0)=\left\{x^{i} \in \mathcal{X}^{i} \mid x^{i} \leq w^{i}\right\}$ and hence by Assumption $\mathrm{U}$ that $d^{i}(p,-2 w, 0)=w^{i}$, implying that markets clear and thus a no-trade equilibrium with full rationing on demand is obtained.

Q.E.D.

The existence of non-trivial equilibria follows from the next result saying that there is a connected set $C$ of QCEs containing both the trivial supply-constrained equilibrium and the trivial demand-constrained equilibrium.

\section{Theorem 3.2}

Let $\mathcal{E}=\left(\left\{\mathcal{X}^{i}, \succeq^{i}, w^{i}\right\}_{i=1}^{m}, P\right)$ be an economy satisfying Assumptions $X, U, W$ and $P$. Then there exists a connected set of Quantity Constrained Equilibria of the economy $\mathcal{E}$, containing a trivial supply-constrained equilibrium $\left(p,-w, w^{1}, \ldots, w^{m}\right)$ for any $p \in P^{0}$, and a trivial demand-constrained equilibrium $\left(p, w, w^{1}, \ldots, w^{m}\right)$ for any $p \in P^{1}$.

We continue this section with the proof of Theorem 3.2. We first focus on the equilibrating mechanism to find a QCE. To do so, we introduce a set $Q \subset \mathbb{R}^{n}$ containing $P$ and define for every $q \in Q$ a price $p(q) \in P$ and a rationing vector $r(q) \in[-w, w]$. The set $Q$ is taken to be

$$
Q=\left\{q \in \mathbb{R}^{n} \mid\|q-p\|_{2} \leq 1 \text { for some } p \in P\right\},
$$

so $Q$ is the set of elements in $\mathbb{R}^{n}$ lying at most at a distance 1 from $P$, using the Euclidean norm, and thus includes the set $P$. In the sequel bnd $(Q)$ denotes the boundary of $Q$ and $\operatorname{int}(Q)$ its interior. Further, for $q \in Q$, define the projection $p(q)$ of $q$ on $P$ by

$$
p(q)=\arg \min _{p \in P}\|p-q\|_{2}
$$


Since by Assumption $\mathrm{P}$, the set $P$ is convex and compact, for every $q \in Q$ it holds that $p(q)$ is uniquely defined and continuous in $q$, and $q-p(q) \in G(p(q))$. Moreover, $\|q-p(q)\|_{2} \leq 1$, with equality if and only if $q \in \operatorname{bnd}(Q)$. It holds that $p(q)=q$ when $q \in P$. The set $Q$ has the following properties.

\section{Lemma 3.3}

(i) The set $Q$ is a convex, compact, full-dimensional subset of $\mathbb{R}^{n}$ and contains $P$ in its interior;

(ii) The boundary of $Q$ is smooth.

Proof. The compactness follows from the compactness of $P$. That $Q$ is full-dimensional and contains $P$ in its interior follows immediately from its definition. To prove convexity, take any $q^{1}, q^{2} \in Q$ and $0 \leq \lambda \leq 1$, and let $q(\lambda)=\lambda q^{1}+(1-\lambda) q^{2}$ and $p(\lambda)=\lambda p\left(q^{1}\right)+(1-\lambda) p\left(q^{2}\right)$. Since $P$ is convex, we know that $p(\lambda) \in P$. Moreover, $\|q(\lambda)-p(\lambda)\|_{2} \leq \lambda\left\|q^{1}-p\left(q^{1}\right)\right\|_{2}+$ $(1-\lambda)\left\|q^{2}-p\left(q^{2}\right)\right\|_{2} \leq 1$. Therefore, $q(\lambda) \in Q$.

Property (ii) follows from the use of the 2-norm in the definition of $Q$. Take any $q^{*} \in \operatorname{bnd}(Q)$. Then $\left\|q^{*}-p\left(q^{*}\right)\right\|_{2}=1$ and any $q$ with $\left\|q-p\left(q^{*}\right)\right\|_{2} \leq 1$ belongs to $Q$. Hence, the normal cone at $q^{*}$ contains at most one vector with length one. Since $Q$ is convex, we know that the normal cone at $q^{*}$ is non-empty and upper-semicontinuous. Hence, the normal cone at any boundary point $q$ of $Q$ contains a unique vector with length one and this vector is continuous in $q$, i.e. the boundary of $Q$ is smooth.

Q.E.D.

We define the function $r: Q \rightarrow \mathbb{R}^{n}$ by

$$
r(q)=0^{n}, \quad q \in P
$$

and, for $k \in I_{n}$,

$$
r_{k}(q)=\left(\min _{j \in I_{n}} \frac{w_{j}}{\left|q_{j}-p_{j}(q)\right|}\right)\left(q_{k}-p_{k}(q)\right)\|q-p(q)\|_{2}, \quad q \in Q \backslash P .
$$

Since $\|q-p(q)\|_{2}$ goes to zero when $q-p(q)$ goes to $0^{n}$ and $-w_{k} \leq\left(\min _{j \in I_{n}} \frac{w_{j}}{\left|q_{j}-p_{j}(q)\right|}\right)\left(q_{k}-\right.$ $\left.p_{k}(q)\right) \leq w_{k}$, it follows that $r(q)$ goes to $0^{n}$ when $q$ converges to a point in $P$. This implies that $r(q)$ is continuous in $q$. The other properties below immediately follow from Lemma 3.3 .

\section{Corollary 3.4}

The function $r: Q \rightarrow \mathbb{R}^{n}$ satisfies the following properties:

(i) $r$ is continuous;

(ii) $-w_{k} \leq r_{k}(q) \leq w_{k}$ for all $q \in Q$;

(iii) $r_{k}(q)=w_{k}$ if and only if $\frac{w_{k}}{q_{k}-p_{k}(q)}=\min _{j \in I_{n}} \frac{w_{j}}{\left|q_{j}-p_{j}(q)\right|}$ and $q \in \operatorname{bnd}(Q)$; 
(iv) $r_{k}(q)=-w_{k}$ if and only if $-\frac{w_{k}}{q_{k}-p_{k}(q)}=\min _{j \in I_{n}} \frac{w_{j}}{\left|q_{j}-p_{j}(q)\right|}$ and $q \in \operatorname{bnd}(Q)$;

(v) $r(q) \in G(p(q))$ for all $q \in Q$.

We now define for any consumer $i \in I_{m}$ the reduced budget correspondence $B^{i}: Q \rightarrow \mathbb{R}^{n}$ by

$$
B^{i}(q)=B^{i}(p(q),-w-r(q), w-r(q)), \quad q \in Q .
$$

Finally, for any consumer $i \in I_{m}$ we define the reduced demand correspondence $d^{i}: Q \rightarrow$ $\mathbb{R}^{n}$ by

$$
d^{i}(q)=\left\{x^{i} \in B^{i}(q) \mid x^{i} \succeq^{i} y^{i}, \text { for all } y^{i} \in B^{i}(q)\right\} .
$$

Because the set $P$ is in the interior of $\mathbb{R}_{+}^{n}$, we have that for any $q \in Q$ the price vector $p(q) \in$ $P$ is strictly positive. Notice that since $-w-r(q)$ can be equal to zero, the cheaper point assumption that is usually required to show continuity of the budget correspondence, is violated. Nevertheless, it follows from Theorem 2.2 in Herings (1996b) that $B^{i}$ is continuous at any $q \in Q$. With Assumption $\mathrm{U}$ it then follows that $d^{i}$ is a continuous function and so is the reduced excess demand function $z: Q \rightarrow \mathbb{R}^{n}$ defined by

$$
z(q)=\sum_{i \in I_{m}} d^{i}(q)-w
$$

By Assumption $\mathrm{U}$ the budget constraint $p(q) \cdot d^{i}(q) \leq p(q) \cdot w^{i}$ is always satisfied with equality and hence Walras' law holds, i.e. $p(q) \cdot z(q)=0$ for all $q \in Q$. The function $z$ satisfies the following properties.

Lemma 3.5 Under Assumptions $X, U, W$ and $P$, the reduced excess demand function $z: Q \rightarrow \mathbb{R}^{n}$ satisfies the following:

(i) $z$ is continuous;

(ii) Walras' law holds: $p(q) \cdot z(q)=0$ for all $q \in Q$;

(iii) $r_{k}(q)=-w_{k}$ implies $z_{k}(q) \geq 0$ and $r_{k}(q)=w_{k}$ implies $z_{k}(q) \leq 0$.

The next theorem shows that a zero point of $z$ on $Q$ induces a QCE.

\section{Theorem 3.6}

Let $q^{*}$ be a zero point of $z$ on $Q$, i.e. $z\left(q^{*}\right)=0^{n}$. Then $\left(p\left(q^{*}\right), r\left(q^{*}\right), d^{1}\left(q^{*}\right), \ldots, d^{m}\left(q^{*}\right)\right)$ is a Quantity Constrained Equilibrium.

\section{Proof.}

We have to show that Conditions (i), (ii) and (iii) of Definition 2.3 hold. Clearly, Conditions (i) and (ii) hold by construction of the reduced excess demand function. By property 
(v) of Corollary 3.4 we have that $r\left(q^{*}\right)$ is an element of $G\left(p\left(q^{*}\right)\right)$, which shows that Condition (iii) of Definition 2.3 holds.

Q.E.D.

From Theorem 3.6 it follows that the question of existence of a QCE reduces to the existence of a zero point of $z$. Before stating our general result on the connected set of constrained equilibria in terms of the parameter $q$, we first show that $Q$ contains two zero points that yield trivial supply-constrained and demand-constrained equilibria. Let $Q^{0}$ and $Q^{1}$ be given by

$$
Q^{0}=\{q \in Q \mid w \cdot q \leq w \cdot \widetilde{q} \text { for all } \tilde{q} \in Q\}
$$

and

$$
Q^{1}=\{q \in Q \mid w \cdot q \geq w \cdot \widetilde{q} \text { for all } \widetilde{q} \in Q\}
$$

Clearly, since $Q$ is compact, the sets $Q^{0}$ and $Q^{1}$ are both non-empty. Moreover, the intersection of $Q^{0}$ and $Q^{1}$ is empty, since $Q$ is full-dimensional.

\section{Theorem 3.7}

Each element in $Q^{0}$ or $Q^{1}$ is a zero point of $z$ and yields a trivial equilibrium:

(i) Any $q \in Q^{0}$ induces the trivial supply-constrained $Q C E\left(p(q),-w, w^{1}, \ldots, w^{m}\right)$ with $p(q) \in P^{0}$.

(ii) Any $q \in Q^{1}$ induces the trivial demand-constrained $Q C E\left(p(q), w, w^{1}, \ldots, w^{m}\right)$ with $p(q) \in P^{1}$.

\section{Proof.}

Take any $q \in Q^{0}$. Clearly, $q \in \operatorname{bnd}(Q)$. By definition of $Q^{0}$ we have that $r(q)=-w$ and $p(q) \in P^{0}$. It follows that, for any $i \in I_{m}, B^{i}(q)=\left\{w^{i}\right\}$ and hence $d^{i}(q)=w^{i}$, implying that $z(q)=0^{n}$ and thus $q$ induces a no-trade equilibrium with full rationing on supply.

Analogously, take any $q \in Q^{1}$. Then $r(q)=w$ and $p(q) \in P^{1}$. It follows that, for any $i \in I_{m}, B^{i}(q)=\left\{x^{i} \in \mathcal{X}^{i} \mid x^{i} \leq w^{i}\right\}$ and hence by Assumption $\mathrm{U}$ that $d^{i}(q)=w^{i}$, implying that $z(q)=0^{n}$ and thus $q$ induces a no-trade equilibrium with full rationing on demand.

Q.E.D.

The existence of non-trivial equilibria follows from the next result saying that $Q$ contains a connected set $C$ of zero points of $z$ having a non-empty intersection with both $Q^{0}$ and $Q^{1}$, i.e. there is a set $C \subset Q$ of zeroes of $z$ being a connected subset of $Q$ satisfying $C \cap Q^{0} \neq \emptyset$ and $C \cap Q^{1} \neq \emptyset$. This implies that there exist a trivial supply constrained equilibrium and a trivial demand constrained equilibrium, which are connected to each other through a set of QCEs. 


\section{Theorem 3.8}

Let $\mathcal{E}=\left(\left\{\mathcal{X}^{i}, \succeq^{i}, w^{i}\right\}_{i=1}^{m}, P\right)$ be an economy satisfying Assumptions $X, U, W$ and $P$. Then there exists a connected set $C \subset Q$ of zero points of $z$ such that $C \cap Q^{0} \neq \emptyset$ and $C \cap Q^{1} \neq \emptyset$.

\section{Proof.}

Let $\gamma_{0}$ and $\gamma_{1}$ be such that $\sum_{k=1}^{n} w_{k} q_{k}=\gamma_{0}$ when $q \in Q^{0}$ and $\sum_{k=1}^{n} w_{k} q_{k}=\gamma_{1}$ when $q \in Q^{1}$. Notice that $\gamma_{0}$ and $\gamma_{1}$ are well-defined, $\gamma_{1}>\gamma_{0}$ and $\gamma_{0} \leq \sum_{k=1}^{n} w_{k} q_{k} \leq \gamma_{1}$ for all $q \in Q$. For some $M>0$, define $X^{0} \subset \mathbb{R}^{n}$ by

$$
X^{0}=\left\{x \in \mathbb{R}^{n} \mid \sum_{k=1}^{n} w_{k} x_{k}=\gamma_{0}, \max _{k \in I_{n}} x_{k} \leq M\right\}
$$

and, for $0<\alpha \leq 1, X^{\alpha} \subset \mathbb{R}^{n}$ by

$$
X^{\alpha}=\left\{x \in \mathbb{R}^{n} \mid x=x^{0}+\frac{\alpha}{\sum_{k=1}^{n} w_{k}^{2}}\left(\gamma_{1}-\gamma_{0}\right) w, x^{0} \in X^{0}\right\} .
$$

Clearly, for $x \in X^{\alpha}, 0 \leq \alpha \leq 1$, we have that $\sum_{k=1}^{n} w_{k} x_{k}=(1-\alpha) \gamma_{0}+\alpha \gamma_{1}$, and thus $\sum_{k=1}^{n} w_{k} x_{k}=\gamma_{0}$ when $x \in X^{0}$ and $\sum_{k=1}^{n} w_{k} x_{k}=\gamma_{1}$ when $x \in X^{1}$. Further, define

$$
X=\cup_{\alpha \in[0,1]} X^{\alpha}
$$

and take $M$ sufficiently large that $Q \subset X$ and that any $q \in Q \backslash\left(Q^{0} \cup Q^{1}\right)$ lies in the interior of $X$. Notice that $Q^{0}=Q \cap X^{0}$ and $Q^{1}=Q \cap X^{1}$.

We define the set $\bar{X}^{0}=\left\{x \in \mathbb{R}^{n} \mid \sum_{k=1}^{n} w_{k} x_{k}=\gamma_{0}\right\}$. Let $\tau(x)$ be the orthogonal projection of $x \in \mathbb{R}^{n}$ on $\bar{X}^{0}$, i.e. $\tau(x)=x-\lambda w$ for some $\lambda \in \mathbb{R}$. Next, let the set $\widetilde{X}^{0}$ be defined by

$$
\widetilde{X}^{0}=\left\{x \in \bar{X}^{0} \mid x=\tau(q+z(q)), q \in Q\right\} \cup\left\{x \in \bar{X}^{0} \mid x=\tau(p(q)), q \in \operatorname{bnd}(Q)\right\} .
$$

Since $Q$ is compact and $z$ and $\tau$ are continuous functions, it follows that $\widetilde{X}^{0}$ is a bounded subset of $\bar{X}^{0}$ and thus $M$ can be taken so large that $X^{0}$ contains $\widetilde{X}^{0}$ in its relative interior.

For $x \in X^{0}$ and $\alpha \in[0,1]$, denote $x^{\alpha}=x+\frac{\alpha}{\sum_{k=1}^{n} w_{k}^{2}}\left(\gamma_{1}-\gamma_{0}\right) w \in X^{\alpha}$ and define the point-to-set mapping $\varphi: X^{0} \times[0,1] \rightarrow X^{0}$ by

$$
\varphi(x, \alpha)=\left\{\begin{array}{l}
\left\{\tau\left(x^{\alpha}+z\left(x^{\alpha}\right)\right)\right\} \text { if } x^{\alpha} \in \operatorname{int}(Q), \\
\operatorname{Conv}\left(\left\{\tau\left(x^{\alpha}+z\left(x^{\alpha}\right)\right)\right\} \cup\left\{\tau\left(p\left(x^{\alpha}\right)\right)\right\}\right) \text { if } x^{\alpha} \in \operatorname{bnd}(Q), \\
\left\{\tau\left(p\left(q\left(x^{\alpha}\right)\right)\right)\right\} \text { if } x^{\alpha} \in X \backslash Q
\end{array}\right.
$$

for all $(x, \alpha) \in X^{0} \times[0,1]$, where Conv(.) denotes the convex hull of a set and $q(x)$ is the orthogonal projection of $x \in \mathbb{R}^{n}$ on $Q$. Clearly, $X^{0}$ is compact and convex, the mapping $\varphi$ is upper semi-continuous, and for every $(x, \alpha) \in X^{0} \times[0,1]$ it holds that $\varphi(x, \alpha)$ is compact, convex and non-empty. According to Browder's theorem, see Browder (1960), there exists 
a connected set $\bar{C}$ of fixed points of $\varphi$ in $X^{0} \times[0,1]$ such that $\bar{C} \cap\left(X^{0} \times\{0\}\right) \neq \emptyset$ and $\bar{C} \cap\left(X^{0} \times\{1\}\right) \neq \emptyset$, i.e. there exists a connected set $\bar{C}$ in $X^{0} \times[0,1]$ satisfying

$$
x \in \varphi(x, \alpha) \text {, for all }(x, \alpha) \in \bar{C},
$$

containing a point in $X^{0} \times\{0\}$, and containing a point in $X^{0} \times\{1\}$. Hence, the set $C \subset X$ defined by

$$
C=\left\{\bar{x} \in X \mid \bar{x}=x^{\alpha},(x, \alpha) \in \bar{C}\right\}
$$

is a connected set in $X$ such that $C \cap X^{0} \neq \emptyset$ and $C \cap X^{1} \neq \emptyset$. It remains to be shown that every element of $C$ lies in $Q$ and is a zero point of $z$.

Take any $\bar{x} \in C$, then $\bar{x}=x^{\alpha}$ for some $(x, \alpha) \in \bar{C}$. Suppose first that $x^{\alpha} \in X \backslash Q$. From the definition of $\varphi$ it then follows that

$$
x=\tau\left(p\left(q\left(x^{\alpha}\right)\right)\right) .
$$

Since $\tau(\bar{x})$ is the projection of $\bar{x}$ on $X^{0}$, there exists $\lambda \in \mathbb{R}$ such that $x=\tau\left(p\left(q\left(x^{\alpha}\right)\right)\right)=$ $p\left(q\left(x^{\alpha}\right)\right)-\lambda w$. Since $x^{\alpha}-q\left(x^{\alpha}\right)=\beta\left(q\left(x^{\alpha}\right)-p\left(q\left(x^{\alpha}\right)\right)\right)$ for some $\beta>0$, it follows that

$$
\begin{aligned}
(\beta+1)\left(q\left(x^{\alpha}\right)-p\left(q\left(x^{\alpha}\right)\right)\right) & =x^{\alpha}-p\left(q\left(x^{\alpha}\right)\right) \\
& =x^{\alpha}-(\lambda w+x) \\
& =\left(\frac{\alpha}{\sum_{k=1}^{n} w_{k}^{2}}\left(\gamma_{1}-\gamma_{0}\right)-\lambda\right) w .
\end{aligned}
$$

This implies that $q\left(x^{\alpha}\right)-p\left(q\left(x^{\alpha}\right)\right)=\delta w$ for some $\delta \in\left\{-\frac{1}{\|w\|_{2}}, \frac{1}{\|w\|_{2}}\right\}$. Hence, either $\alpha=0$ and $x^{\alpha} \in Q^{0}$, or $\alpha=1$ and $x^{\alpha} \in Q^{1}$. This contradicts that $x^{\alpha} \in X \backslash Q$. Consequently, $x^{\alpha} \in Q$ for every $x^{\alpha} \in C$.

Next, suppose that $x^{\alpha} \in \operatorname{int}(Q)$. Then $x^{\alpha}-\frac{\alpha}{\sum_{k=1}^{n} w_{k}^{2}}\left(\gamma_{1}-\gamma_{0}\right) w=x=\tau\left(x^{\alpha}+z\left(x^{\alpha}\right)\right)$. Since $\tau\left(x^{\alpha}+z\left(x^{\alpha}\right)\right)=x^{\alpha}+z\left(x^{\alpha}\right)-\lambda w$ for some $\lambda \in \mathbb{R}$, it follows that

$$
z\left(x^{\alpha}\right)=\left(\lambda-\frac{\alpha}{\sum_{k=1}^{n} w_{k}^{2}}\left(\gamma_{1}-\gamma_{0}\right)\right) w .
$$

Because of Walras' law, we obtain

$$
p\left(x^{\alpha}\right) \cdot z\left(x^{\alpha}\right)=\left(\lambda-\frac{\alpha}{\sum_{k=1}^{n} w_{k}^{2}}\left(\gamma_{1}-\gamma_{0}\right)\right) p\left(x^{\alpha}\right) \cdot w=0 .
$$

Hence, since $p\left(x^{\alpha}\right) \cdot w>0$, we have that $\lambda=\frac{\alpha}{\sum_{k=1}^{n} w_{k}^{2}}\left(\gamma_{1}-\gamma_{0}\right)$ and thus $z\left(x^{\alpha}\right)=0^{n}$, showing that $x^{\alpha}$ is a zero point of $z$.

Finally, suppose $x^{\alpha} \in \operatorname{bnd}(Q)$. Then there exists $\beta_{1} \geq 0$ and $\beta_{2} \geq 0$ with $\beta_{1}+\beta_{2}=1$ such that

$$
\beta_{1} \tau\left(x^{\alpha}+z\left(x^{\alpha}\right)\right)+\beta_{2} \tau\left(p\left(x^{\alpha}\right)\right)=x=x^{\alpha}-\frac{\alpha}{\sum_{k=1}^{n} w_{k}^{2}}\left(\gamma_{1}-\gamma_{0}\right) w .
$$


Using that $\tau(\bar{x})$ is the projection of $\bar{x}$ on $X^{0}$, it follows that this can be rewritten as

$$
\beta_{1} x^{\alpha}+\beta_{1} z\left(x^{\alpha}\right)+\beta_{2} p\left(x^{\alpha}\right)-\mu w=x^{\alpha}-\frac{\alpha}{\sum_{k=1}^{n} w_{k}^{2}}\left(\gamma_{1}-\gamma_{0}\right) w
$$

for some $\mu \in \mathbb{R}$. With $\beta_{1}+\beta_{2}=1$ and letting $\lambda=\mu-\frac{\alpha}{\sum_{k=1}^{n} w_{k}^{2}}\left(\gamma_{1}-\gamma_{0}\right)$ we obtain

$$
\beta_{1} z\left(x^{\alpha}\right)=\beta_{2}\left(x^{\alpha}-p\left(x^{\alpha}\right)\right)+\lambda w .
$$

First, suppose $\beta_{1}=0$. Then, as before, it follows that $x^{\alpha}-p\left(x^{\alpha}\right)=\delta w$ for some $\delta \in$ $\left\{-\frac{1}{\|w\|_{2}}, \frac{1}{\|w\|_{2}}\right\}$, implying that $x^{\alpha} \in Q^{0} \cup Q^{1}$. Hence, according to Theorem 3.7, $z\left(x^{\alpha}\right)=0^{n}$ and thus $x^{\alpha}$ is a zero point of $z$. Second, suppose $\beta_{1}>0$. Then

$$
z\left(x^{\alpha}\right)=\frac{\beta_{2}}{\beta_{1}}\left(x^{\alpha}-p\left(x^{\alpha}\right)\right)+\frac{\lambda}{\beta_{1}} w=\frac{\delta}{\beta_{1}} r\left(x^{\alpha}\right)+\frac{\lambda}{\beta_{1}} w,
$$

for some $\delta \geq 0$. Since $x^{\alpha} \in \operatorname{bnd}(Q)$, we have that there is $k$ such that $\left|r_{k}\left(x^{\alpha}\right)\right|=w_{k}$. When $r_{k}\left(x^{\alpha}\right)=-w_{k}$ it follows that

$$
z_{k}\left(x^{\alpha}\right)=-\frac{\delta}{\beta_{1}} w_{k}+\frac{\lambda}{\beta_{1}} w_{k} \geq 0,
$$

so $\lambda \geq \delta$. Hence, $-\delta+\lambda>0$ and thus

$$
z_{h}\left(x^{\alpha}\right)=\frac{\delta}{\beta_{1}} r_{h}\left(x^{\alpha}\right)+\frac{\lambda}{\beta_{1}} w_{h} \geq-\frac{\delta}{\beta_{1}} w_{h}+\frac{\lambda}{\beta_{1}} w_{h} \geq 0
$$

for all $h \in I_{n}$ and therefore $z\left(x^{\alpha}\right)=0^{n}$ due to Walras' law. Similarly, when $r_{k}\left(x^{\alpha}\right)=w_{k}$ it follows that

$$
z_{k}\left(x^{\alpha}\right)=\frac{\delta}{\beta_{1}} w_{k}+\frac{\lambda}{\beta_{1}} w_{k} \leq 0,
$$

for some $\delta \geq 0$ and thus $\delta+\lambda \leq 0$. Then

$$
z_{h}\left(x^{\alpha}\right)=\frac{\delta}{\beta_{1}} r_{h}\left(x^{\alpha}\right)+\frac{\lambda}{\beta_{1}} w_{h} \leq \frac{\delta}{\beta_{1}} w_{h}+\frac{\lambda}{\beta_{1}} w_{h} \leq 0
$$

for all $h \in I_{n}$ and therefore $z\left(x^{\alpha}\right)=0^{n}$ due to Walras' law.

Q.E.D.

The theorem guarantees that there is a connected set of QCEs connecting two trivial equilibria. Each QCE in this set is induced by some $q^{*} \in C$ and is equal to $\left(p^{*}, r^{*}, x^{*}\right)=$ $\left(p\left(q^{*}\right), r\left(q^{*}\right), x\left(q^{*}\right)\right)$, where $x\left(q^{*}\right)$ is the demand allocation induced by $q^{*}$. One trivial equilibrium is induced by some $q^{0} \in Q^{0}$ and the other one by some $q^{1} \in Q^{1}$. At $q^{0}$ we have $r\left(q^{0}\right)=-w$ and so $p\left(q^{0}\right) \in P^{0}$, full rationing on the supply and no rationing on the demand. At $q^{1}$ we have $r\left(q^{1}\right)=w$ and so $p\left(q^{1}\right) \in P^{1}$, no rationing on supply, and full rationing on demand. Hence, at $p^{0}=p\left(q^{0}\right)$ the prices within $P$ cannot be decreased further in the direction $-w$, i.e. $-w$ is in the normal cone $G\left(p^{0}\right)$, and this vector $-w$ determines the rationing scheme $\left(\ell^{0}, u^{0}\right)$ with $\ell^{0}=0^{n}$ and $u^{0} \geq w$. Similarly, at $p^{1}=p\left(q^{1}\right)$, the prices within $P$ 
cannot be increased further in the direction $w$, and this vector determines the full demand rationing scheme $\left(\ell^{1}, u^{1}\right)$ where $u^{1}=0^{n}$ and $\ell^{1}=-2 w$. A non-trivial QCE is induced by any $q \in C$ not in $Q^{0}$ or $Q^{1}$. If $q \in P$, then $p(q)=q$ is a Walrasian equilibrium price vector, $r(q)=0^{n}$, and the induced rationing scheme given by $(\ell, u)=(-w, w)$ is not binding, i.e. no consumer is rationed in his demand or supply of any of the goods. If $q$ is not in $P$ and so $p(q) \neq q$, then $p(q)$ lies on the boundary of the set $P$ of admissible prices, the vector $q-p(q)$ lies in the gradient $G(p(q))$ at $p(q)$ to $P$ and so the prices at $p(q)$ cannot be moved further in the direction $q-p(q)$ without leaving $P$. We are now ready to prove Theorem 3.2 .

\section{Proof of Theorem 3.2}

Take the function $f: Q \rightarrow P \times \mathbb{R}^{n} \times \prod_{i \in I_{m}} \mathcal{X}^{i}$ defined by

$$
f(q)=\left(p(q), r(q), x^{1}(q), \ldots, x^{m}(q)\right), \quad q \in Q .
$$

Consider the connected set $C$ of zero points of $z$ as defined in Theorem 3.8. It follows by Theorem 3.6 that any point in $C$ induces a QCE of $\mathcal{E}$. Since $f$ is continuous and $C$ is connected by Theorem 3.8, it follows that $f(C)$ is connected. By Theorem 3.7, it follows that $f(q)$ is a trivial supply-constrained equilibrium whenever $q \in Q^{0}$ and $f(q)$ is a trivial demand-constrained equilibrium whenever $q \in Q^{1}$. Since $C$ intersects both $Q^{0}$ and $Q^{1}, f(C)$ contains both a trivial supply-constrained equilibrium and a trivial demandconstrained equilibrium.

Q.E.D.

Having proved that there exists a connected set of QCEs containing both a trivial supplyconstrained equilibrium and a trivial demand-constrained equilibrium, it is easy to show that this set contains several elements inducing QCEs with some specific properties. These properties are most easily stated in terms of the parametrization by the set $Q$, with each $q \in Q$ inducing a price $p(q)$, a rationing vector $r(q)$, and an allocation $\left(x^{1}(q), \ldots, x^{m}(q)\right)$.

First of all, notice that for any $q^{0} \in C \cap Q^{0}$ it holds that $r\left(q^{0}\right)=-w$ and that for any $q^{1} \in C \cap Q^{1}$ it holds that $r\left(q^{1}\right)=w$. Therefore, since $C$ connects $Q^{0}$ and $Q^{1}$ and $r$ is a continuous function on $Q$, for any a priori chosen $k \in I_{n}$ there exists a $q(k) \in C$ satisfying $r_{k}(q(k))=0$. Such a $q(k)$ induces a rationing scheme $(\ell, u)$ with $\ell_{k}=-w_{k}$ and $u_{k}=w_{k}$. The point $q(k)$ in $Q$ therefore induces a QCE at which no consumer is being rationed in commodity $k$. We call such an equilibrium a generalized Drèze equilibrium with respect to commodity $k$.

\section{Corollary 3.9}

For any $k \in I_{n}$, there exists a generalized Drèze equilibrium with respect to commodity $k$.

The corollary implies that if commodity $k$ is the numeraire, there exists a QCE at which the numeraire is not being rationed. Similarly, there exists a $q^{-} \in C$ satisfying $\max _{j} r_{j}\left(q^{-}\right)=0$. 
This element of $C$ induces a QCE at which at least one commodity is not rationed and all other commodities are rationed only in their supply and therefore is a generalized supply-constrained equilibrium. Similarly, there exists a QCE at which consumers are only rationed in their demand and at least one commodity is not rationed, a generalized demand-constrained equilibrium. Such an equilibrium is induced by a point $q^{+}$in $C$ for which $\min _{j} r_{j}\left(q^{+}\right)=0$. Notice that both types of equilibria are QCEs at which at least one commodity is not rationed, but it cannot be said in advance which commodity is not rationed.

\section{Corollary 3.10}

There exists a generalized supply-constrained equilibrium and there exists a generalized demand-constrained equilibrium.

\section{Applications and extensions}

Let us consider the case that $P$ is a ball with center a given price system $\widehat{p}$. Without loss of generality, we assume that $\widehat{p}_{k}>1$ for any $k \in I_{n}$ and that the radius of the ball is 1 , so that any price vector in the set is strictly positive. Therefore the set of admissible prices $P$ is given by

$$
B=\left\{p \in \mathbb{R}^{n} \mid\|p-\widehat{p}\|_{2} \leq 1\right\} .
$$

Clearly, the set $Q$ is a ball around $\widehat{p}$ with radius 2 and for any $q \in Q$ it holds that $r(q)=0^{n}$ if $q \in B$ and $r(q)=\mu(q)(p(q)-\hat{p})$ for some positive number $\mu(q)$ when $q \in Q \backslash B$. Moreover, for any $p \in \operatorname{bnd}(P)$, we have that $G(p)=\left\{r \in \mathbb{R}^{n} \mid r=\lambda(p-\widehat{p}), \lambda \geq 0\right\}$. Then both sets $Q^{0}=\left\{\hat{p}-\frac{2}{\|w\|_{2}} w\right\}$ and $Q^{1}=\left\{\hat{p}+\frac{2}{\|w\|_{2}} w\right\}$ contain one element. From Theorem 3.8 it follows that there exists a connected set of zero points of $z$ connecting the unique element $q^{0}=\widehat{p}-\frac{2}{\|w\|_{2}} w$ of $Q^{0}$ with the unique element $q^{1}=\widehat{p}+\frac{2}{\|w\|_{2}} w$ of $Q^{1}$. Hence, there exists a connected set of QCEs connecting the unique trivial equilibrium with full supply rationing and price vector $\widehat{p}-\frac{1}{\|w\|_{2}} w$ induced by $q^{0}$ with the unique trivial equilibrium with full demand rationing and price vector $\hat{p}+\frac{1}{\|w\|_{2}} w$ induced by $q^{1}$. According to Corollary 3.9 this connected set contains a generalized Drèze equilibrium with respect to commodity $k$ for any $k \in I_{n}$. In case the point $q(k)$ inducing this equilibrium does not belong to $B$, it must hold that $r_{k}(q(k))=0$ and $r(q(k))=\mu_{k}(p(q(k))-\widehat{p})$ for some $\mu_{k}>0$, implying that the price $p_{k}(q(k))$ of commodity $k$ must be precisely equal to $\hat{p}_{k}$. In case $q(k)$ lies in $B$, $q(k)$ is a Walrasian price system. Summarizing we have the following corollary.

\section{Corollary 4.1}

Suppose $P$ is a ball of positive prices with center $\hat{p}$ and radius 1 . Then there exist a 
connected set of QCEs connecting the unique trivial supply-constrained equilibrium with price $\hat{p}-\frac{1}{\|w\|_{2}} w$ to the unique trivial demand-constrained equilibrium with price $\hat{p}+\frac{1}{\|w\|_{2}} w$. The connected set contains for every $k \in I_{n}$ a generalized Drèze equilibrium with respect to $k$. Such a Drèze equilibrium is a Walrasian equilibrium or has $p_{k}=\widehat{p}_{k}$.

As a second example we consider the case

$$
P=\left\{p \in \mathbb{R}_{+}^{n} \mid \underline{p}_{j} \leq p_{j} \leq \bar{p}_{j}, j \in I, \underline{\pi}_{j}\left(p_{I}\right) \leq p_{j} \leq \bar{\pi}_{j}\left(p_{I}\right), j \in J\right\}
$$

where $I \subset I_{n}$ is a set of index commodities and $J=I_{n} \backslash I$ is the set of indexed commodities with prices bounded from below and above by index functions depending on the prices of the commodities in $I$. This set generalizes the set of admissible prices considered in Dehez and Drèze (1984) and satisfies the so-called noncircularity condition of Weddepohl (1987), saying that the prices may not be indexed directly or indirectly by themselves. We assume that for any $j \in I$ it holds that $0<\underline{p}_{j} \leq \bar{p}_{j}<\infty$ and that for any $j \in J$, the lower bound function $\underline{\pi}_{j}$ is convex, the upper bound function $\bar{\pi}_{j}$ is concave and that for all feasible $p_{I}$ it holds that $0<\underline{\pi}_{j}\left(p_{I}\right) \leq \bar{\pi}_{j}\left(p_{I}\right)<\infty$, so that $P$ satisfies Assumption P. To give a characterization of a QCE, for simplicity we assume that all index functions are continuously differentiable.

Suppose $q \in Q$ induces a QCE and thus $z(q)=0^{n}$. Since $r(q) \in G(p(q))$, we have that $p(q) \cdot r(q)=\max _{p \in P} p \cdot r(q)$. From the first-order Kuhn-Tucker conditions it then follows that there exists nonnegative numbers $\underline{\lambda}_{j}$ and $\bar{\lambda}_{j}, j \in I_{n}$, such that

$$
\begin{aligned}
& r_{j}(q)=\bar{\lambda}_{j}-\underline{\lambda}_{j}+\sum_{h \in J} \underline{\lambda}_{h} \frac{\partial \underline{\pi}_{h}\left(p_{I}(q)\right)}{\partial p_{j}}-\sum_{h \in J} \bar{\lambda}_{h} \frac{\partial \bar{\pi}_{h}\left(p_{I}(q)\right)}{\partial p_{j}}, j \in I, \\
& r_{j}(q)=\bar{\lambda}_{j}-\underline{\lambda}_{j}, j \in J
\end{aligned}
$$

and

$$
\begin{aligned}
& \underline{\lambda}_{j}\left(p_{j}(q)-\underline{p}_{j}\right)=0, \bar{\lambda}_{j}\left(\bar{p}_{j}-p_{j}(q)\right)=0, \underline{\lambda}_{j} \bar{\lambda}_{j}=0, j \in I, \\
& \underline{\lambda}_{j}\left(p_{j}(q)-\underline{\pi}_{j}\left(p_{I}(q)\right)\right)=0, \bar{\lambda}_{j}\left(\bar{\pi}_{j}\left(p_{I}(q)\right)-p_{j}(q)\right)=0, \underline{\lambda}_{j} \bar{\lambda}_{j}=0, j \in J .
\end{aligned}
$$

Recall that in a QCE Condition (iii) of Definition 2.3 is satisfied, but not necessarily Condition (iv) of Definition 2.1. So, when a commodity is rationed in its supply (demand), its price is not necessarily on its lower (upper) bound. To make this more precise, first take an indexed commodity $j \in J$. From (4) and the last complementarity condition in (6) it follows that

$$
\underline{\lambda}_{j}=-r_{j}(q)>0 \text { if } r_{j}(q)<0 \text {, and } \bar{\lambda}_{j}=r_{j}(q)>0 \text { if } r_{j}(q)>0 \text {. }
$$


It follows with the first two complementarity conditions in $(6)$ that $p_{j}(q)=\underline{\pi}_{j}\left(p_{I}(q)\right)$ if commodity $j \in J$ is rationed in its supply and that $p_{j}(q)=\bar{\pi}_{j}\left(p_{I}(q)\right)$ if commodity $j \in J$ is rationed in its demand, i.e. in case of supply (demand) rationing the price of an indexed commodity is on its lower (upper) bound given the prices $p_{I}(q)$ of the index commodities. For an index commodity $j \in I$, in general such a clear link does not exist. However, suppose that all index functions are monotonically increasing in $p_{I}$ and all the derivatives are positive. Now, if $p_{k}(q)>\underline{\pi}_{k}\left(p_{I}(q)\right)$ for all $k \in J$, then $\underline{\lambda}_{k}=0$ for all $k \in J$ and it follows from equation (3) that for all $j \in I$ it holds

$$
r_{j}(q)=\bar{\lambda}_{j}-\underline{\lambda}_{j}-\sum_{k \in J} \bar{\lambda}_{k} \frac{\partial \bar{\pi}_{k}\left(p_{I}(q)\right)}{\partial p_{j}} \leq \bar{\lambda}_{j}-\underline{\lambda}_{j},
$$

and thus $\bar{\lambda}_{j}>0$ if $r_{j}(q)>0$. Hence, if the prices of the indexed commodities are all above their lower bound, rationing on the demand of an index commodity can only occur when its price is on its upper bound. Clearly, when the price of an indexed commodity is on its lower bound, demand rationing of an index commodity may occur, caused by the fact that the prices of the indexed commodities cannot be decreased further. Similarly, if the prices of the indexed commodities are all below their upper bound, rationing on the supply of an index commodity can only occur when its price is on its lower bound. In particular, at a trivial supply-constrained (demand-constrained) equilibrium the prices of all the indexed commodities are on their lower (upper) bound given $p_{I}(q)$, and thus also the prices of all the index commodities are on their lower (upper) bounds. Summarizing we have the following corollary.

\section{Corollary 4.2}

Let $P$ be given as in (2) and let $q \in Q$ be such that $z(q)=0^{n}$. Then, for all $j \in J$,

$$
p_{j}(q)=\underline{\pi}_{j}\left(p_{I}(q)\right) \text { if } r_{j}(q)<0 \text { and } p_{j}(q)=\bar{\pi}_{j}\left(p_{I}(q)\right) \text { if } r_{j}(q)>0 .
$$

When all index functions are monotonically increasing, then, for all $j \in I$,

$$
p_{j}(q)=\underline{p}_{j} \text { if } r_{j}(q)<0 \text { and for all } k \in J, p_{k}(q)<\bar{\pi}_{k}\left(p_{I}(q)\right),
$$

and

$$
p_{j}(q)=\bar{p}_{j} \text { if } r_{j}(q)>0 \text { and for all } k \in J, p_{k}(q)>\underline{\pi}_{k}\left(p_{I}(q)\right) \text {. }
$$

Next we consider an extension allowing that the set of admissible prices is unbounded. We show that there is an unbounded connected set of QCEs with at least one of the prices going to infinity. Let $P$ be a convex, closed, unbounded set of admissible prices in the interior of $\mathbb{R}_{+}^{n}$ and define $Q=\left\{q \in \mathbb{R}^{n} \mid\|q-p\|_{2} \leq 1\right.$, for some $\left.p \in P\right\}$. Notice that $Q^{0}$ is 
non-empty, while $Q^{1}$ is empty. For $q \in Q$, let $p(q), r(q)$, and $z(q)$ be defined as before. Let $\gamma_{0}=\sum_{k=1}^{n} w_{k} q_{k}$ for any $q \in Q^{0}$, define for $s \geq \gamma_{0}$ the set $Q(s)$ by

$$
Q(s)=\left\{q \in Q \mid \sum_{k=1}^{n} w_{k} q_{k} \leq s\right\},
$$

and for any $s>\gamma_{0}, \bar{Q}(s)=\left\{q \in Q(s) \mid \sum_{k=1}^{n} w_{k} q_{k}=s\right\}$ as the upper boundary of $Q(s)$. Applying Theorem 3.8 to $Q(s)$ and using the fact that $Q(s) \cap X^{0}=Q^{0}$ and $Q(s) \cap X^{1}=$ $\bar{Q}(s)$, it follows that there exists a connected set of zero points of $z$ in $Q(s)$ containing a point in $Q^{0}$ and a point in $\bar{Q}(s)$ for any $s>s_{0}$. Clearly, a zero point in $Q^{0}$ induces again a trivial supply-constrained equilibrium. However, in general a zero point in the upper boundary $\bar{Q}(s)$ is not a trivial demand-constrained equilibrium. The next theorem says that there exists an unbounded connected set of zero points of $z$ in $Q$ containing a point in $Q^{0}$.

Theorem 4.3 Let $\mathcal{E}=\left(\left\{\mathcal{X}^{i}, \succeq^{i}, w^{i}\right\}_{i=1}^{m}, P\right)$ be an economy satisfying Assumptions $X, U$ and $W$, and let $P$ be a non-empty, convex, closed, unbounded set in the interior of $\mathbb{R}_{+}^{n}$. Then there exists an unbounded connected subset $C$ of $Q$ such that $C \cap Q^{0} \neq \emptyset$ and each point in $C$ is a zero point of the reduced excess demand function $z$.

\section{Proof}

For $s>s_{0}$, let $C(s)$ be a connected set of zero points of $z$ in $Q(s)$ containing a point in $Q^{0}$ and a point in $\bar{Q}(s)$. Take $C=Q^{0} \cup\left(\cup_{s>s_{0}} C(s)\right)$. Since any point in $Q^{0}$ is a zero point of $z$, the set $C$ is an unbounded connected set of zero points of $z$ in $Q$ such that $C \cap Q^{0} \neq \emptyset$.

Q.E.D.

As a specific example we consider, for some arbitrarily small $\varepsilon>0$, the set $P$ given by

$$
P=\left\{p \in \mathbb{R}_{+}^{n} \mid p_{j} \geq \varepsilon, j \in M, \sum_{j \in I} p_{j}=1, \underline{p}_{j} \leq p_{j} \leq \bar{p}_{j}, j \in I \cup J\right\},
$$

where $M, I$ and $J$ form a partition of $I_{n}{ }^{2}$ This set generalizes sets of admissible prices considered in Kurz (1982), van der Laan (1984) and Weddepohl (1987), where $M$ is the set of numeraire commodities whose prices can move freely above $\varepsilon, I$ is a set of index commodities with sum of the prices normalized to one, and $J$ is a set of indexed commodities with lower and upper bounds on the prices. We assume that for any $j \in I \cup J$ it holds that $0<\underline{p}_{j} \leq \bar{p}_{j}<\infty$ and $\sum_{j \in I} \underline{p}_{j}<1<\sum_{j \in I} \bar{p}_{j}$. Without loss of generality, we may assume that $I=\{1, \ldots,|I|\}$ and $w_{j+1} \geq w_{j}, j=1, \ldots|I|-1$. Moreover, for the sake of simplicity, we restrict ourselves to the generic case that there is no partition $I^{1}, I^{2}$ of $I$

\footnotetext{
${ }^{2}$ For simplicity we take prices bounded away from zero. The analysis can be extended to $\varepsilon=0$ by generalizing the concept of constrained equilibrium to quasi-constrained equilibrium as has been discussed in Kurz (1982).
} 
such that $\sum_{j \in I^{1}} \underline{p}_{j}+\sum_{j \in I^{2}} \bar{p}_{j}=1$. Then, let $j^{\prime} \in I$ be such that $\sum_{j=1}^{j^{\prime}} \underline{p}_{j}+\sum_{j=j^{\prime}+1}^{|I|} \bar{p}_{j}<1$ and $\sum_{j=1}^{j^{\prime}-1} \underline{p}_{j}+\sum_{j=j^{\prime}}^{|I|} \bar{p}_{j}>1$.

Suppose $q \in Q$ induces a QCE and thus $z(q)=0^{n}$. Again, $r(q) \in G(p(q))$ implies $p(q) \cdot r(q)=\max _{p \in P} p \cdot r(q)$. From the first-order Kuhn-Tucker conditions it then follows that there exist nonnegative numbers $\lambda_{j}, j \in M$, nonnegative numbers $\underline{\lambda}_{j}, \bar{\lambda}_{j}, j \in I \cup J$, and a number $\lambda_{I}$ such that

$$
\begin{aligned}
& r_{j}(q)=-\lambda_{j}, j \in M, \\
& r_{j}(q)=\lambda_{I}-\underline{\lambda}_{j}+\bar{\lambda}_{j}, j \in I, \\
& r_{j}(q)=-\underline{\lambda}_{j}+\bar{\lambda}_{j}, j \in J,
\end{aligned}
$$

and furthermore the following complementarity conditions hold

$$
\begin{aligned}
& \lambda_{j}\left(p_{j}(q)-\varepsilon\right)=0, j \in M, \\
& \underline{\lambda}_{j}\left(p_{j}(q)-\underline{p}_{j}\right)=0, \bar{\lambda}_{j}\left(\bar{p}_{j}-p_{j}(q)\right)=0, \underline{\lambda}_{j} \bar{\lambda}_{j}=0, j \in I \cup J
\end{aligned}
$$

At a trivial supply-constrained equilibrium we have that $r(q)=-w$, so that $\lambda_{j}=w_{j}$ and hence $p_{j}(q)=\varepsilon$ for all $j \in M, \underline{\lambda}_{j}=w_{j}, \bar{\lambda}_{j}=0$ and hence $p_{j}(q)=\underline{p}_{j}$ for all $j \in J$, while for $j \in I$ it holds

$$
\lambda_{I}-\underline{\lambda}_{j}+\bar{\lambda}_{j}=-w_{j}
$$

We claim that $\lambda_{I}=-w_{j^{\prime}}$, for all $j=1, \ldots, j^{\prime}-1$ it holds that $\underline{\lambda}_{j}=0$ and $\bar{\lambda}_{j}=w_{j^{\prime}}-w_{j} \geq 0$, $\underline{\lambda}_{j^{\prime}}=\bar{\lambda}_{j^{\prime}}=0$, and for all $j=j^{\prime}+1, \ldots,|I|$ it holds that $\bar{\lambda}_{j}=0$ and $\underline{\lambda}_{j}=w_{j}-w_{j^{\prime}} \geq 0$. It then follows immediately that prices of commodities $j=1, \ldots, j^{\prime}-1$ satisfy $p_{j}=\bar{p}_{j}$, and prices of commodities $j=j^{\prime}+1, \ldots,|I|$ satisfy $p_{j}=\underline{p}_{j}$. Intuitively, from the properties that $p(q) \cdot r(q)=\max _{p \in P} p \cdot r(q), r(q)=-w$ and $\sum_{j \in I} p_{j}=1$, it follows that the prices of the commodities with the larger total endowments are set on their minimum level, and the prices of commodities with the smaller total endowments on their maximum level.

To formally show our claim, consider a commodity $k \in\{1, \ldots,|I|\}=I$ such that $\underline{p}_{k}<p_{k}(q)<\bar{p}_{k}$. Such a commodity $k$ exists, since we consider the case that there is no partition $I^{1}, I^{2}$ of $I$ such that $\sum_{j \in I^{1}} \underline{p}_{j}+\sum_{j \in I^{2}} \bar{p}_{j}=1$. It follows immediately that $\underline{\lambda}_{k}=\bar{\lambda}_{k}=0$, so $\lambda_{I}=-w_{k}$. Consider any commodity $j \in\{1, \ldots, k-1\}$. Then it follows from equation (9) that $\underline{\lambda}_{j}-\bar{\lambda}_{j}=\lambda_{I}-r_{j}(q)=\lambda_{I}+w_{j}=-w_{k}+w_{j} \leq 0$, so that $\underline{\lambda}_{j}=0$ and $\bar{\lambda}_{j}=w_{k}-w_{j} \geq 0$. Similarly, for any commodity $j \in\{k+1, \ldots,|I|\}$ it follows from equation (9) that $\underline{\lambda}_{j}-\bar{\lambda}_{j}=-w_{k}+w_{j} \geq 0$, so that $\bar{\lambda}_{j}=0$ and $\underline{\lambda}_{j}=-w_{k}+w_{j} \geq 0$. Together with the complementarity properties given in (12) it follows that

$$
\sum_{j=1}^{k-1} p_{j}(q)+p_{k}(q)+\sum_{j=k+1}^{|I|} p_{j}(q)=\sum_{j=1}^{k-1} \bar{p}_{j}+p_{k}(q)+\sum_{j=k+1}^{|I|} \underline{p}_{j}=1 .
$$


Therefore,

$$
\begin{gathered}
\sum_{j=1}^{k} \bar{p}_{j}+\sum_{j=k+1}^{|I|} \underline{p}_{j} \geq 1, \\
\sum_{j=1}^{k-1} \bar{p}_{j}+\sum_{j=k}^{|I|} \underline{p}_{j} \leq 1,
\end{gathered}
$$

so $k=j^{\prime}$. Summarizing, at a trivial supply-constrained equilibrium, the prices of all the numeraire and indexed commodities are on its lower bound, while the price of precisely one index commodity is not bounded and the prices of the index commodities with larger total endowments are set on their minimum level, and the prices of the index commodities with smaller total endowments on their maximum level.

From the equations (8)-(12) it follows that at any QCE it holds that a numeraire commodity can only be rationed in its supply, which only occurs when its price is $\varepsilon$, while an indexed commodity can only be rationed in its supply (demand) when its price is on its lower (upper) bound. For an index commodity $j \in I$ rationing can also occur when its price is not binding, due to the restriction that the sum of the prices of the index commodities is equal to one. For all commodities $j \in I$ with non-binding prices we have that $r_{j}(q)=\lambda_{I}$. Therefore, all these commodities are either not rationed $\left(\lambda_{I}=0\right)$, or rationed with the same amount on either their demands $\left(\lambda_{I}>0\right)$ or their supplies $\left(\lambda_{I}<0\right)$. At a QCE induced by a point $q$ satisfying $\sum_{j \in I} q_{j}=1$, it holds that $\lambda_{I}=0$ and a commodity in $I$ can only be rationed in its supply (demand) if its price is on its lower (upper) bound. The existence of such a point follows as a corollary from the next theorem.

Theorem 4.4 Let $\mathcal{E}=\left(\left\{\mathcal{X}^{i}, \succeq^{i}, w^{i}\right\}_{i=1}^{m}, P\right)$ be an economy satisfying Assumptions $X, U$, $W$ and let the set $P$ be as specified in (7). Then there exists an $\bar{s}>s_{0}$ such that for all zero points $q \in Q$ of $z$ with $\sum_{k=1}^{n} q_{k} \geq \bar{s}$ it holds that $r_{j}(q)=w_{j}$ for all $j \in I \cup J, p_{j}(q)>\varepsilon$ and $r_{j}(q)=0$ for all $j \in M$.

\section{Proof.}

From Theorem 4.3 it follows that there exists a sequence $\left(q^{t}\right)_{t \in \mathbf{N}}$ in $Q$, satisfying $\sum_{h=1}^{n} q_{h}^{t} \geq t$ and $z\left(q^{t}\right)=0^{n}$ for all $t \in \mathbb{N}$. Since $P$ and thus also $Q$ is bounded in any $j \in I \cup J$, without loss of generality it follows that $q_{k}^{t} \rightarrow \infty$ for some $k \in M$. It is obvious that $r_{k}\left(q^{t}\right)=0$. Now, define $I^{t}=\left\{j \in I \mid r_{j}\left(q^{t}\right)<w_{j}\right\}, J^{t}=\left\{j \in J \mid r_{j}\left(q^{t}\right)<w_{j}\right\}$ and $M^{t}=\left\{j \in M \mid p_{j}\left(q^{t}\right)=\varepsilon\right\}$. Then, for any $j \in I \cup J \cup M^{t}$ we have that $\frac{p_{j}\left(q^{t}\right)}{p_{k}\left(q^{t}\right)}$ goes to zero. Moreover, for all $i \in I_{m}$ and $j \in I_{n}$, the demand $d_{j}^{i}\left(q^{t}\right)$ is bounded from above by $w_{j}^{i}+\left(w_{j}-r_{j}\left(q^{t}\right)\right)$, while $r_{k}\left(q^{t}\right)=0$ implies net supply equal to $-w_{k}$ is possible for commodity $k$. For $t$ sufficiently large, for any $i \in I_{m}$, for any $j \in I^{t} \cup J^{t} \cup M^{t}$, it follows from the monotonicity of the preferences that $d_{j}^{i}\left(q^{t}\right)=w_{j}^{i}+\left(w_{j}-r_{j}\left(q^{t}\right)\right)>w_{j}^{i}$, contradicting $z\left(q^{t}\right)=0^{n}$. Hence $I^{t} \cup J^{t} \cup M^{t}=\emptyset$. 
So, for $t$ sufficiently large it follows that $r_{j}\left(q^{t}\right)=w_{j}$ and that $p_{j}\left(q^{t}\right)>\varepsilon$ and $r_{j}\left(q^{t}\right)=0$ for all $j \in M$.

Q.E.D.

The theorem implies that there exists a connected set of QCEs containing a trivial supplyconstrained equilibrium and an equilibrium with full rationing on the demand of the commodities in $I \cup J$ and no rationing at all on the commodities in $M$ with prices above $\varepsilon$. Clearly, at the latter equilibrium it must hold that for every consumer the consumption of the commodities in $I \cup J$ is equal to its initial endowments of these commodities. Hence the prices of the commodities in $M$ are Walrasian equilibrium prices for a reduced economy with only commodities in $M$ and preferences on this reduced commodity space obtained from the preferences in $\mathcal{E}$ with consumption of the commodities in $I \cup J$ equal to the initial endowments. At any $q^{0}$ inducing a trivial equilibrium we have that $r\left(q^{0}\right)=-w$, while at any $\bar{q}$ inducing an equilibrium with full rationing on the demand of commodities in $I \cup J$ and Walrasian prices for the commodities in $M$, it holds that $r_{j}(\bar{q})=w_{j}$ for all $j \in I \cup J$, and $r_{j}(\bar{q})=0$ for all $j \in M$. Observe that $r_{j}\left(q^{0}\right)=-w_{j}$ for all $j \in I$ implies that for some $\mu^{0}>0$

$$
\sum_{j \in I} q_{j}^{0}=\sum_{j \in I}\left(\mu^{0} r_{j}\left(q^{0}\right)+p_{j}\left(q^{0}\right)\right)=-\mu^{0} \sum_{j \in I} w_{j}+\sum_{j \in I} p_{j}\left(q^{0}\right)=-\mu^{0} \sum_{j \in I} w_{j}+1<1
$$

and that $r_{j}(\bar{q})=w_{j}$ for all $j \in I$ implies that for some $\bar{\mu}>0$

$$
\sum_{j \in I} \bar{q}_{j}=\sum_{j \in I}\left(\bar{\mu} r_{j}(\bar{q})+p_{j}(\bar{q})\right)=\bar{\mu} \sum_{j \in I} w_{j}+\sum_{j \in I} p_{j}(\bar{q})=\bar{\mu} \sum_{j \in I} w_{j}+1>1 .
$$

From this we obtain the following corollary.

Corollary 4.5 Let $\mathcal{E}=\left(\left\{\mathcal{X}^{i}, \succeq^{i}, w^{i}\right\}_{i=1}^{m}, P\right)$ be an economy satisfying Assumptions $X, U$, $W$ and let the set $P$ be as specified in (7). Then there exists a connected set of QCEs, containing a trivial supply-constrained equilibrium and the following types of equilibrium:

(i) For any $j \in I \cup J$, an equilibrium induced by a point $q$ satisfying $r_{j}(q)=0$, i.e. a generalized Drèze equilibrium with respect to commodity $j \in I \cup J$;

(ii) An equilibrium induced by a point $q$ satisfying $r_{j}(q)=0$ for all $j \in M$, i.e. a generalized Drèze equilibrium in which no commodity $j \in M$ is rationed;

(iii) An equilibrium induced by a point $q$ satisfying $\max _{j \in I_{n}} r_{j}(q)=0$, i.e. a supplyconstrained equilibrium, so at least one of the commodities is unconstrained;

(iv) An equilibrium induced by a point $q$ satisfying $\max _{j \in I \cup J} r_{j}(q)=0$, i.e. a supplyconstrained equilibrium in which at least one of the commodities in $I \cup J$ is unconstrained; (v) An equilibrium induced by a point $q$ satisfying $\sum_{j \in I} q_{j}=1$, i.e. a commodity in I can only be rationed on its supply (demand) if its price is on its lower (upper) bound;

(vi) An equilibrium with full rationing on the demands on the commodities in $I \cup J$ and Walrasian prices for the reduced economy with commodities in $M$. 
At an equilibrium of type (iv) it may hold for some $j \in M$ that $r_{j}(q)<0$ and hence supply rationing occurs on some of the commodities in $M$. At such an equilibrium we have that for some $h \in I \cup J, r_{h}(q)=0$ and $p_{h}(q) \geq \underline{p}_{h}>0$ and thus $p_{h}(q)\left(-w_{h}-r_{h}(q)\right)<0$. Now, suppose that $r_{j}(q)<0$ and thus $p_{j}(q)=\varepsilon$ for some $j \in M$. From the monotonicity of the preferences it then follows for sufficiently small $\varepsilon$ that $d_{j}^{i}(q)=w_{j}^{i}+\left(w_{j}-r_{j}(q)\right)>w_{j}^{i}$ for all $i \in I_{m}$, contradicting that $z_{j}(q)=0$. Hence we must have that $r_{j}(q)=0$ for all $j \in M$, implying that all commodities in $M$ are unconstrained.

Corollary 4.6 Let $\mathcal{E}=\left(\left\{\mathcal{X}^{i}, \succeq^{i}, w^{i}\right\}_{i=1}^{m}, P\right)$ be an economy satisfying Assumptions $X, U$, $W$ and let the set $P$ be as specified in (7). Then for $\varepsilon$ sufficiently close to zero, there exists a supply-constrained equilibrium in which none of the commodities in $M$ are rationed and at least one of the commodities in $I \cup J$ is not rationed.

This corollary generalizes the results of van der Laan (1984) and Weddepohl (1987) obtained for the case with one money commodity in $M$ and no lower and upper bounds for the index commodities in $I$. Finally, if $I \cup J=\emptyset$, the equilibrium of type (vi) is a Walrasian equilibrium for the economy $\mathcal{E}$ with price set $P=\left\{p \in \mathbb{R}_{+}^{n} \mid p_{j} \geq \varepsilon, j \in I_{n}\right\}$ and thus in this case there is a connected set of equilibria connecting the trivial no-trade fully supply constrained equilibrium with a Walrasian equilibrium.

\section{References}

[1] K.J. Arrow and G. Debreu (1954), Existence of an equilibrium for a competitive economy, Econometrica, 22, 265-290.

[2] J.-P. Bénassy (1975), Neo-Keynesian disequilibrium theory in a monetary economy, Review of Economic Studies 42, 503-523.

[3] J.-P. Bénassy (1993), Nonclearing markets: microeconomic concepts and macroeconomic applications, Journal of Economic Literature 31, 732-761.

[4] F.E. Browder (1960), On continuity of fixed points under deformation of continuous mappings, Summa Brasilia Mathematica 4, 183-191.

[5] V.K. Chetty and P.R. Nayak (1978), Drèze equilibria for polyhedral and strictly convex price sets, Discussion paper, Indian Statistical Institute, New Delhi, India.

[6] A. Citanna, H. Crès, J. Drèze, P.J.J. Herings, and A. Villanacci (2001), Continua of underemployment equilibria reflecting coordination failures, also at Walrasian prices, METEOR Research Memorandum 01/10, Universiteit Maastricht, pp. 1-33. 
[7] C.C. Cox (1980), The enforcement of public price controls, Journal of Political Economy 88, 887-916.

[8] G. Debreu (1959), Theory of Value, Yale University Press, New Haven, USA.

[9] P. Dehez and J.H. Drèze (1984), On supply-constrained equilibria, Journal of Economic Theory 33, 172-182.

[10] J.H. Drèze (1975), Existence of an exchange economy under price rigidities, International Economic Review 16, 310-320.

[11] J.H. Drèze (1997), Walras-Keynes equilibria, coordination and macroeconomics, European Economic Review 41, 1737-1762.

[12] J.H. Drèze (2001), On the macroeconomics of uncertainty and incomplete markets, Recherches Economiques de Louvain, 67, 1-29.

[13] J.H. Drèze and C. Gollier (1993), Risk sharing on the labour market, European Economic Review, 37, 1457-1482.

[14] V.A. Ginsburgh and L. Van der Heyden (1975), On extending the Negishi approach to computing equilibria: the case of government price support policies, Journal of Economic Theory 44, 168-178.

[15] P.J.J. Herings (1996a), Static and Dynamic Aspects of General Equilibrium Theory, Kluwer Academic Publishers, Dordrecht, The Netherlands.

[16] P.J.J. Herings (1996b), Equilibrium existence results for economies with price rigidities, Economic Theory, 7, 63-80.

[17] P.J.J. Herings (1997), Endogenously determined price rigidities, Economic Theory, 9, 471-498.

[18] P.J.J. Herings (1998), On the existence of a continuum of constrained equilibria, Journal of Mathematical Economics, 30, No. 3, 257-273.

[19] P.J.J. Herings and H.M. Polemarchakis (2000), Pareto improving price regulation when the asset market is incomplete, METEOR Research Memorandum 00/16, Universiteit Maastricht.

[20] P.J.J. Herings, A.J.J. Talman, and Z. Yang (1996), The computation of a continuum of constrained equilibria, Mathematics of Operations Research, 21, 675-696. 
[21] M. Kurz (1982), Unemployment equilibria in an economy with linked prices, Journal of Economic Theory 26, 110-123.

[22] G. van der Laan (1980), Equilibrium under rigid prices with compensation for the consumers, International Economic Review 21, 53-73.

[23] G. van der Laan (1982), Simplicial approximation of unemployment equilibria, Journal of Mathematical Economics 9, 83-97.

[24] G. van der Laan (1984), Supply-constrained fixed price equilibria in monetary economies, Journal of Mathematical Economics 13, 171-187.

[25] T.T. Nguyen and J. Whalley (1986), Equilibrium under price controls with endogenous transactions costs, Journal of Economic Theory 39, 290-300.

[26] T.T. Nguyen and J. Whalley (1990), General equilibrium analysis of price controls: a compuational approach, International Economic Review 31, 667-684.

[27] J. Tuinstra (2000), The emergence of political business cycles in a two-sector general equilibrium model, European Journal of Political Economy, 16, 509-534.

[28] Y. Younès (1975), On the role of money in the process of exchange and the existence of a non-Walrasian equilibrium, Review of Economic Studies, 42, 489-501.

[29] L. Walras (1874), Éléments d'économie politique pure, Corbaz, Lausanne, Switzerland.

[30] C. Weddepohl (1987), Supply-constrained equilibria in economies with indexed prices, Journal of Economic Theory 43, 203-222. 